\title{
Clinical applicability of monitoring pulmonary artery blood flow acceleration time variations in monitoring fetal pulmonary artery pressure
}

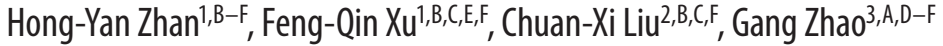 \\ ${ }^{1}$ Department of B-Ultrasound, Fourth People's Hospital of Jinan, China \\ 2 Department of B-Ultrasound, Shandong Provincial Hospital Affiliated to Shandong University, Jinan, China \\ ${ }^{3}$ Department of Emergency Surgery, Shandong Province Qianfoshan Hospital, Shandong University, Jinan, China \\ A - research concept and design; $\mathrm{B}$ - collection and/or assembly of data; $\mathrm{C}$ - data analysis and interpretation; \\ $D$ - writing the article; $E$ - critical revision of the article; $F$ - final approval of the article
}

Address for correspondence

Gang Zhao

E-mail: zhaogang198s@sina.cn

Funding sources

Science and Technology Star Program of Jinan Science and Technology Bureau of Shandong Province (grant No. 201406017).

Conflict of interest None declared

Received on February 7, 2017

Reviewed on April 14, 2017

Accepted on July 5, 2017

\section{Abstract}

Background. In recent years, pulmonary artery blood flow acceleration time (AT) has been believed to be applicable in the examination of fetal lung development.

Objectives. This study aims to evaluate the clinical significance of pulmonary artery blood flow AT as a parameter in monitoring of fetal pulmonary artery pressure.

Material and methods. A total of 31 fetuses in mid- or late-term pregnancy with tricuspid regurgitation were set as the study group (congenital heart disease with a tricuspid regurgitation pressure difference of more than $20 \mathrm{~mm}$ Hg was excluded). A total of $68 \mathrm{normal}$ fetuses in mid- or late-term pregnancy were selected as the control group (strictly screened for tricuspid regurgitation, congenital heart disease and other congenital diseases before inclusion). The average ATs of both groups were calculated. Correlations of pulmonary artery systolic pressure (PASP) and AT, as well as the ratio of AT to right ventricular ejection time (ET) (AT/ET ratio) of both groups were investigated by 1-way analysis of variance (ANOVA).

Results. The average AT of the study group was significantly lower than that of the control group $(p<0.0001)$. In the study group, AT negatively correlated with PASP $(r=-0.52 ; p<0.01)$, AT/ET ratio negatively correlated with PASP $(r=-0.52 ; p<0.01)$ and both showed statistical significance.

Conclusions. The results indicated that fetuses in the study group showed lower ATs and AT/ET ratios than the control group. Acceleration times and AT/ET ratios decreased as PASP increased. Thus, AT and AT/ET ratio can be used clinically as new parameters for the qualitative and - to some extent - quantitative evaluation of fetal pulmonary artery pressure.

Key words: pulmonary artery systolic pressure, fetal ultrasonic cardiogram, pulmonary artery blood flow acceleration time

DOI

$10.17219 /$ acem $/ 75686$

Copyright

Copyright by Author(s)

This is an article distributed under the terms of the

Creative Commons Attribution Non-Commercial License

(http://creativecommons.org/licenses/by-nc-nd/4.0/) 


\section{Introduction}

In recent years, Schenone et al. have introduced the determination of pulmonary artery blood flow acceleration time (AT) into the research on the development of fetal pulmonary tissues for the first time. ${ }^{1}$ Acceleration time is believed to be applicable to the examination of fetal lung development. Kim et al. have managed to examine neonatal respiratory distress syndrome. ${ }^{2}$ Previous studies have reported an association of AT and AT/ejection time (ET) ratio with the Doppler flow curve of the fetal pulmonary artery. ${ }^{3-5}$ Till now, no report has been published on the association between AT and pulmonary artery pressure. The present study is an investigation on using AT and $\mathrm{AT} / \mathrm{ET}$ ratio as new parameters for monitoring fetal pulmonary artery pressure, based on the study of the AT of fetuses in mid- or late-term pregnancy with simple high pulmonary pressure.

\section{Material and methods}

\section{Patients}

Information and ultrasonic cardiograms from the regular fetal ultrasonic cardiogram examinations of over 26,000 pregnant women, carried out between June 2011 and December 2013, were collected from the Ultrasonography Department of Provincial Hospital, the Maternal and Child Health Center of Jinan, the Ultrasonography Department of the Fourth People's Hospital of Jinan, and Maternity and Child Healthcare Hospital of Shizhong District, Jining (China) (Table 1).

\section{Trial grouping}

Study group consisted of 31 subjects and included 8 mid-term pregnant women aged $28 \pm 4$ years (gestational age: $26 \pm 1$ weeks) and 23 late-term pregnant women aged $28 \pm 4$ years (gestational age: $33 \pm 5$ weeks). The inclusion criteria were: 1 . enlarged right atrium or ventricle; 2 . medium or severe tricuspid regurgitation; 3. tricuspid regurgitation pressure difference $<20 \mathrm{~mm} \mathrm{Hg}$; and 4 . no tricuspid regurgitation induced by vascular deformity in congenital heart disease.

The control group consisted of 68 subjects, including 33 mid-term pregnant women aged $27 \pm 5$ years (gestational age: $26 \pm 1$ weeks) and 35 late-term pregnant women aged $29 \pm 6$ years (gestational age: $33 \pm 5$ weeks). None of the pregnant women habitually used tobacco or alcohol, and none of them had a history of chronic diseases, including pulmonary tuberculosis or rheumatic heart diseases. Their fetuses were normal according to physical examination; fetal deformity screening was strictly conducted to exclude heart deformity. The inclusion criteria were: 1. normal cardiac cavity sizes; 2 . no tricuspid regurgitation; and 3. no vascular malformation caused by congenital heart diseases.

\section{Methods of ultrasonographic examination}

"Fetal heart" mode was selected. The standard sections of fetal ultrasonic cardiograms recommended by the American Society of Echocardiography were employed, and a regular fetal examination was conducted by a cranial side-deviated 4-chamber view. ${ }^{6}$ The pregnant women assumed a supine or lateral position. At first, the position of the fetal heart was detected, then the angles of examination and other parameters (depth, gain, focus, etc.) were optimized based on the conditions of the mother and the baby in order to obtain a standard and clear long-axis view of the pulmonary artery. ${ }^{7}$ The pulsed-waved Doppler (PW) sampling spot was set at $3 \mathrm{~mm}$ over the pulmonary valve. A recording rate of $100 \mathrm{~cm} / \mathrm{s}$ was selected for better accuracy and blood spectrum performance. Acceleration time (Fig. 1A) and ET were recorded, and AT/ET ratio was calculated. Acceleration time is the time interval from the start of blood flow spectrum of the right ventricle contraction phase to the time-point of max contraction velocity. Ejection time is the time interval from the start of right ventricular contraction to the end of right ventricular contraction. In a standard 4-chamber view, the max velocity of tricuspid regurgitation and the pressure difference were determined by continuous-wave Doppler (CW) (Fig. 1B). All data was presented as the average value of 3 measurements. The angle between the directions of blood flow and the acoustic beam was kept within $20^{\circ}$ to assure the accuracy of blood flow velocity and the pressure difference.

\section{Calculation of pulmonary artery systolic pressure}

Pulmonary artery systolic pressure (PASP) is the right ventricular pressure (Fig. 1A) + the tricuspid regurgitation

Table 1. Subjects' characteristics

\begin{tabular}{|c|c|c|c|c|c|}
\hline Group & Pregnancy type & $\mathrm{n}$ & $\begin{array}{l}\text { Average age } \\
\text { [years] }\end{array}$ & $\begin{array}{l}\text { Range of gestational age } \\
\text { [weeks] }\end{array}$ & $\begin{array}{c}\text { Average gestational age } \\
\text { [weeks] }\end{array}$ \\
\hline \multirow{2}{*}{ Study group } & Mid-term pregnancy & 8 & $28 \pm 4$ & $24-27$ & $26 \pm 1$ \\
\hline & Late-term pregnancy & 23 & $28 \pm 4$ & $28-39$ & $33 \pm 5$ \\
\hline \multirow{2}{*}{ Control group } & Mid-term pregnancy & 33 & $27 \pm 5$ & $26-27$ & $26 \pm 1$ \\
\hline & Late-term pregnancy & 35 & $29 \pm 6$ & $28-38$ & $33 \pm 5$ \\
\hline
\end{tabular}



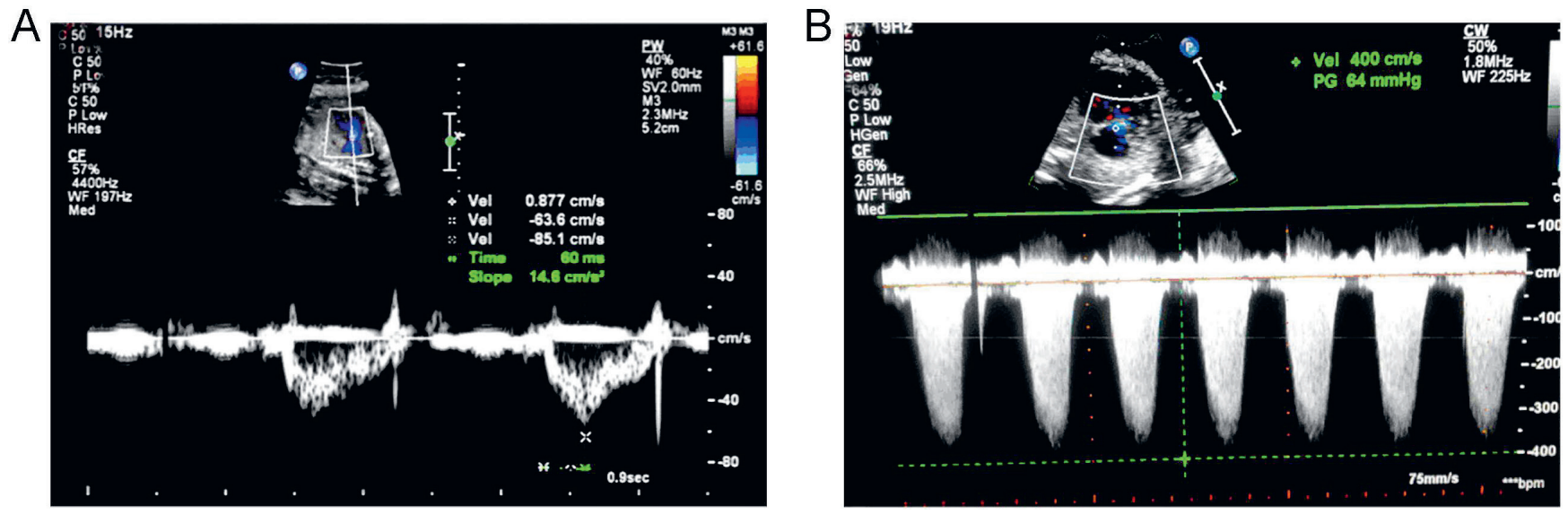

Fig. 1. Blood flow spectrum and tricuspid regurgitation

A - blood flow spectrum of the fetus (AT was from the start to the peak); B - determination of the max velocity of tricuspid regurgitation and the pressure difference; AT - acceleration time

pressure difference (Fig. 1B). The right ventricular pressure of the fetuses was calculated based on the right ventricular pressure of an adult $(5 \mathrm{~mm} \mathrm{Hg}$ in mild tricuspid regurgitation, $10 \mathrm{~mm} \mathrm{Hg}$ in moderate tricuspid regurgitation and $15 \mathrm{~mm} \mathrm{Hg}$ in severe tricuspid regurgitation) and was set as follows: $3 \mathrm{~mm} \mathrm{Hg}$ in mild tricuspid regurgitation, $6 \mathrm{~mm} \mathrm{Hg}$ in moderate tricuspid regurgitation and $11 \mathrm{~mm} \mathrm{Hg}$ in severe tricuspid regurgitation. Further, the tricuspid regurgitation pressure difference was considered to be $2 \mathrm{~mm} \mathrm{Hg}$ in mild tricuspid regurgitation, $3 \mathrm{~mm} \mathrm{Hg}$ in moderate tricuspid regurgitation and $5 \mathrm{~mm} \mathrm{Hg}$ in severe tricuspid regurgitation.

The severity of tricuspid regurgitation was graded as follows: none (grade 0), no regurgitant jet; mild (grade 1+), a jet area $<20 \%$ in the right atrial area; moderate (grade $2+)$, a jet area of $20-33 \%$ in the right atrial area; and severe (grade $3+$ ), a jet area $>33 \%$ in the right atrial area.

\section{Statistical analysis}

SPSS v. 17.0 (SPSS Inc., Chicago, USA) was employed in the statistical analysis; all values were presented as mean \pm standard deviation (SD). Student's t-test was used in intergroup comparison. Multiple factors of the observation group were analyzed with bivariate correlation. Pearson's correlation coefficient was verified by t-statistics. A p-value $<0.05$ was considered statistically significant.

\section{Results}

The average AT of the study group was $47.6 \pm 5.0 \mathrm{~ms}$, whereas the average AT of the control group was $54.3 \pm 9.8 \mathrm{~ms}$, and the difference was very significant $(p<0.0001)$.
The average AT/ET ratio of the study group was $0.24 \pm 0.02$, the average AT/ET ratio of the control group was $0.27 \pm 0.04$ and the difference was statistically significant $(\mathrm{p}<0.01)$ (Table 2).

The results indicated that AT negatively correlated with PASP ( $\mathrm{r}=-0.52 ; \mathrm{p}<0.01$ ) (Fig. 2); AT/ET ratio negatively correlated with PASP $(\mathrm{r}=-0.52 ; \mathrm{p}<0.01)$ (Fig. 3).

The results indicated that the AT values of the fetuses with increased pulmonary artery pressure were significantly lower than those of normal fetuses. Meanwhile, AT decreased as pulmonary artery pressure increased.

\section{Discussion}

In 1987, the negative relationship between pulmonary artery pressure and pulmonary artery blood flow AT was discovered in the correlative analysis on the pulmonary artery blood flow AT of adults and the average pulmonary artery pressure determined by cardiac catheter, conducted by Dabestani et al., where an increase in pulmonary artery pressure resulted in shortened pulmonary artery blood flow AT. ${ }^{6}$ Average pulmonary artery pressure was $0.79-(0.45 \times \mathrm{AT}) \mathrm{mm} \mathrm{Hg}$, based on the reported data. ${ }^{7}$ As reported by Chaoui et al., pulmonary hypertension in adults can lead to increased pulmonary vascular resistance, showing an abnormal blood flow spectrum similar to the aorta; in their study, AT was shortened and the peak appeared earlier, and the extent of increase correlated to the severity of pulmonary hypertension. ${ }^{8}$ Kitabatake et al. revealed that when average pulmonary artery pressure was $<20 \mathrm{~mm} \mathrm{Hg}$ in an adult, AT was $137 \pm 24 \mathrm{~ms}$, and when average pulmonary artery

Table 2. Comparison of ATs and AT/ET ratios of the study group and the control group of mid- and late-term pregnancy fetuses

\begin{tabular}{|l|c|c|}
\multicolumn{1}{|c|}{ Index } & Study group & Control group \\
\hline Pulmonary artery blood flow AT [ms] & $47.6 \pm 5.0$ & $54.3 \pm 9.8$ \\
\hline Ratio of AT and right ventricular blood ET (AT/ET) & $0.24 \pm 0.02$ & $<0.01$ \\
\hline
\end{tabular}




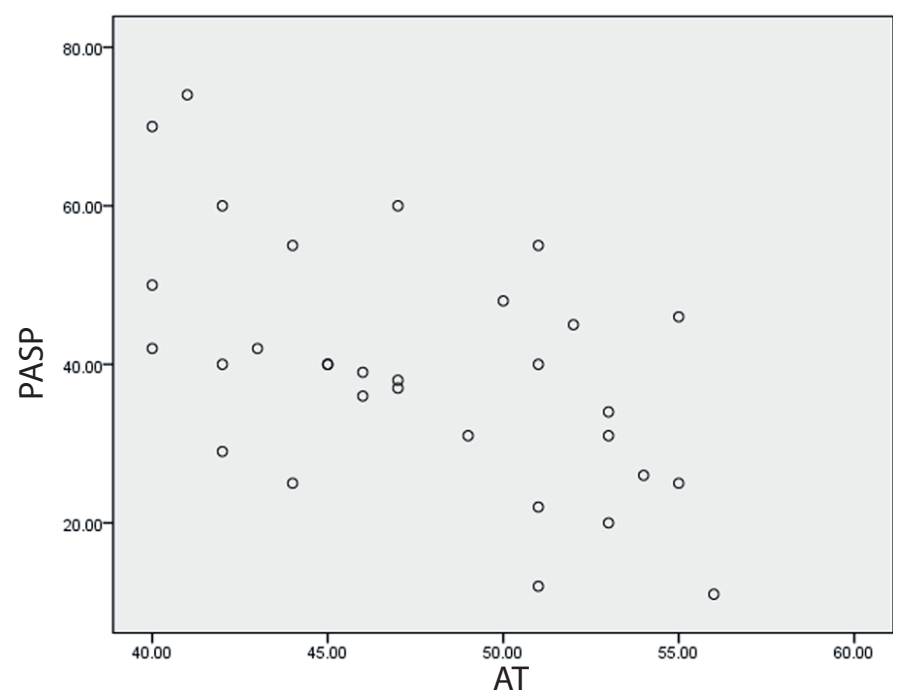

Fig. 2. A negative correlation was shown in the correlation analysis of AT and PASP $(r=-0.52 ; p<0.01)$

AT - acceleration time; PASP - pulmonary artery systolic pressure.

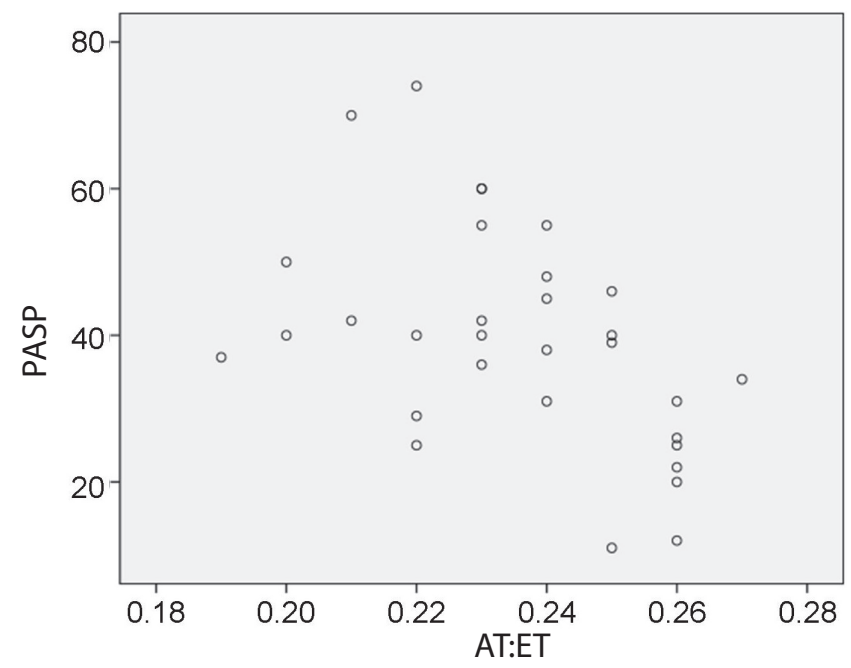

Fig. 3. A negative correlation was shown in the correlation analysis of AT/ET ratio and PASP $(r=-0.52 ; p<0.01)$

AT - acceleration time; ET - ejection time; PASP - pulmonary artery systolic pressure.

pressure was $>20 \mathrm{~mm} \mathrm{Hg}$, AT was $97 \pm 20 \mathrm{~ms}^{9}{ }^{9}$ Nevertheless, the accuracy of pulmonary artery pressure determination can be affected by the operator's expertise, the selection of sections, and the angle between the sonic beam and blood flow. Granstam et al. have elucidated the clinical significance of the determination of blood AT in the systolic phase at the pulmonary valve in the classification of pulmonary hypertension. ${ }^{10}$ Acceleration times of 70-90 ms, 50-70 ms and $<50 \mathrm{~ms}$ corresponded to mild, medium and severe pulmonary hypertension, respectively. Kim et al. prognosed neonatal respiratory distress syndrome using pulmonary artery AT and AT/ET ratio as parameters, and their research proved that $\mathrm{AT}$ was a prospectively useful index in the prognosis of neonatal respiratory distress syndrome, but no correlative study was included on pulmonary artery pressure. ${ }^{2}$ Pavankumar et al. reported the significance of mid- to late-term functional pulmonary hypertension; they revealed that a pressure difference in severe tricuspid regurgitation in fetuses $>70 \mathrm{~mm} \mathrm{Hg}$ indicated that PASP of the fetus can be $>70 \mathrm{~mm} \mathrm{Hg}$, in which case right ventricular failure may occur, leaving the fetus with a potentially fatal outcome if elective premature delivery is not conducted. ${ }^{11}$ All the abovementioned reports have shown that studies on pulmonary artery pressure from different points of view are drawing more and more attention in clinical practice.

As discovered through years of clinical practice, tricuspid regurgitation is very common among fetuses in mid- or late-term pregnancy; mostly this is physiological regurgitation, and the incidence is approx. 6.0\%. ${ }^{12,13}$ Pathological or high-speed regurgitation accounts for about $0.12 \%$. The possible explanations for this condition are likely to be the increased resistance along with the growth of the fetus and the compressed vascular cavity, strengthened by the contractility of the artery or resistance in pulmonary arteries. Increased pulmonary artery pressure can lead to increased systolic pressure of the right ventricle, and eventually to tricuspid regurgitation. The higher pulmonary artery pressure, the greater regurgitation volume. Severe pulmonary hypertension and tricuspid regurgitation can result in right ventricular failure manifested by an increased ventricular rate, a significantly enlarged right heart, or signs such as pericardium, peritoneal or pleural effusion. Therefore, it is critical to monitor the pulmonary artery pressure in the etiological analysis of right heart enlargement and tricuspid regurgitation in fetuses. In the fetal period, the only determination method for pulmonary artery pressure is non-invasive Doppler ultrasonography. ${ }^{14}$ Currently, the commonly used method is to calculate the right ventricular systolic pressure by adding the right ventricular pressure to the measured value of tricuspid regurgitation pressure difference; the right ventricular systolic pressure is equal to PASP when no pulmonary stenosis is present. By the same method, pulmonary artery diastolic pressure (PADP) can be calculated from the pulmonary valve regurgitation.

However, in some cases, tricuspid or pulmonary valve regurgitation was too mild to be detected, or difficult to detect due to the position of the fetus. ${ }^{15}$ Determination of the ratio of pulmonary artery blood flow AT and ET is a novel approach for monitoring pulmonary artery pressure. The results of this study indicated that AT was negatively correlated with PASP, and that increased PASP could lead to a decrease in AT. Thus, AT can be used as a more accessible parameter in measuring pulmonary artery pressure. Especially when pulmonary artery pressure increases, and no tricuspid or pulmonary valve regurgitation is detected, AT and AT/ET ratio are more convincing in signifying pulmonary artery pressure.

Acceleration time and AT/ET ratio are semi-quantitative parameters in pulmonary artery pressure determination 
that can be used in the approximate evaluation of pulmonary hypertension; whether "average pulmonary artery pressure $=0.79-(0.45 \times \mathrm{AT}) \mathrm{mm} \mathrm{Hg}$ " - a rule for calculating adult pulmonary pressure - is applicable in the quantitative determination of fetal pulmonary artery pressure, still needs more clinical evidence and more specific research. ${ }^{16}$

Although this study produced some interesting findings, there were also a few limitations. Firstly, the foramina ovale (FO), which has a significant influence on the diameters of right ventricle and right atrium, will finally result in changes in PASP. Therefore, the FO is important for evaluating cardiac function, which should be investigated in further studies. Secondly, the numbers of patients involved in the study group and the control group were relatively small, though over 26,000 pregnant women were examined through regular ultrasound in this study. In future studies, we would involve a larger number of patients.

In conclusion, for fetuses of mid- or late-term pregnancy, AT and AT/ET ratio are closely related to PASP, especially for those showing no signs of tricuspid regurgitation; thus, $\mathrm{AT}$ and AT/ET ratio can be used as qualitative and semi-quantitative parameters in the determination of fetal pulmonary artery pressure, based on which a new method for pulmonary artery pressure determination has been proposed for clinical diagnosis.

\section{References}

1. Schenone MH, Samson JE, Jenkins L, Suhag A, Mari G. Predicting fetal lung maturity using the fetal pulmonary artery Doppler wave acceleration/ejection time ratio. Fetal Diagn Ther. 2014;36(3):208-214. doi: 10.1159/000358299

2. Kim SM, Park JS, Norwitz ER, et al. Acceleration time-to-ejection time ratio in fetal pulmonary artery predicts the development of neonatal respiratory distress syndrome: A prospective cohort study. Am J Perinatol. 2013;30(10):805-812. doi: 10.1055/s-0032-1333132

3. Guan Y, Li S, Luo G, et al. The role of Doppler waveforms in the fetal main pulmonary artery in the prediction of neonatal respiratory distress syndrome. J Clin Ultrasound. 2015;43(6):375-383. doi: 10.1002/ jcu. 22219
4. Rychik J, Ayres N, Cuneo B, et al. American Society of Echocardiography guidelines and standards for performance of the fetal echocardiogram. J Am Soc Echocardiogr. 2004;17(7):803-810.

5. Lindeley W, Hale R, Spear A, et al. Does corticosteroid impact fetal pulmonary artery blood flow in women at risk for preterm birth? Med Ultrason. 2015;17(3):280-283. doi: 10.11152/mu.2013.2066.173.wly

6. Dabestani A, Mahan G, Gardin JM, et al. Evaluation of pulmonary artery pressure and resistance by pulsed Doppler echocardiography. Am J Cardiol. 1987;59(6):662-668.

7. Chuanxi L, Chuiping L. Screening and Diagnosis of Fetal Heart Malformation by Color Doppler Ultrasound. Beijing, China: Scientific and Technical Documents Publishing House; 2003.

8. Chaoui R, Taddei F, Rizzo G, Bast C, Lenz F, Bollmann R. Doppler echocardiography of the main stem of the pulmonary arteries in the normal human fetus. Ultrasound Obstet Gynecol. 1998;11(3):173-179.

9. Kitabatake A, Inoue M, Asao M, et al. Noninvasive evaluation of pulmonary hypertension by a pulsed Doppler technique. Circulation. 1983;68(2):302-309.

10. Granstam SD, Björklund E, Wikström G, Roos MW. Use of echocardiographic pulmonary acceleration time and estimated vascular resistance for the evaluation of possible pulmonary hypertension. Cardiovasc Ultrasound. 2013;11(1):7. doi: 10.1186/1476-7120-11-7

11. Pavankumar $P$, Venugopal $P$, Kaul U, et al. Closed mitral valvotomy during pregnancy: A 20-year experience. Scand J Thorac Cardiovasc Surg. 1988;22(1):11-15.

12. Pawelec M, Dżugalik M, Pietras J, Bełza $Ł$, Latkowski Ł. Medical and ethical considerations related to viable fetuses with trisomy 13 in the $36^{\text {th }}$ week of pregnancy: A review of the literature. Adv Clin Exp Med. 2015;24(5): 911-921. doi: 10.17219/acem/26324

13. DiBardino DJ, Jacobs JP. Current readings: Long-term management of patients undergoing successful pediatric cardiac surgery. Semin Thorac Cardiovasc Surg. 2014;26(2):132-144. doi: 10.1053/j.semtcvs. 2014.08.002

14. Barczykowska E, Szwed-Kolińska M, Wróbel-Bania A, Ślusarz R. The use of central venous lines in the treatment of chronically ill children. Adv Clin Exp Med. 2014;23(6):1001-1009. doi: 10.17219/ acem/37359

15. Chiappa E, Micheletti A, Sciarrone A, Botta G, Abbruzzese P. The prenatal diagnosis of and short-term outcome for patients with congenitally corrected transposition. Cardiol Young. 2004;14(3):265-276.

16. Hansel J, Burgstahler C, Medler S, Axmann D, Niess AM, Tetzlaff K. Effect of simulated diving trips on pulmonary artery pressure in healthy men. Clin Res Cardiol. 2012;101(12):947-953. doi: 10.1007/ s00392-012-0482-9 